\title{
Drei Lieder von Wilhelm Arbenz
}

\author{
Eine Bieler Geschichte
}

Wilhelm Arbenz ist wohl die wichtigste Figur des Bieler Musiklebens im 2o. Jahrhundert. Ohne Unterlass setzte er sich für die musikalische Bildung und die von ihm gegründeten Musikinstitutionen ein. Als am Freitag, 1. September 1939 der Zweite Weltkrieg ausbrach, komponierte Wilhelm Arbenz drei Lieder nach Robert Walser für Sopran und Klavier; am Ende jedes Lieds vermerkte er das Datum. Im Schaffen von Wilhelm Arbenz, das stark auf Laienmusik ausgerichtet war, bildet diese Liedergruppe, was den Kunstanspruch und die kompositorische Qualität angeht, eine große Ausnahme. Es sind wahrscheinlich die ersten Walser-Vertonungen eines Schweizer Komponisten überhaupt.

\subsection{Einleitung}

Zwei Tage bevor Wilhelm Arbenz (1899-1967) die Gedichte Enttäuschung, Trug und Wintersonne vertonte, war Henri Guisan von der Vereinigten Bundesversammlung zum General ernannt und die Teilmobilmachung veranlasst worden. In Biel wurden 4000 Männer aufgeboten, ein Zehntel der damaligen Bieler Bevölkerung. ${ }^{1}$ Paradoxerweise hatte sich die Bieler Uhrenindustrie dank der Produktion von Zeitzündern für die deutsche Wehrmacht von der Wirtschaftskrise erholt und ihre Exportzahlen seit 1935 mehr als verdoppelt; die Bieler Uhrenmacher leisteten sich neue Radioapparate, um Hitlers Reden hören zu können. ${ }^{2}$ Ein Jahr später rettete Wilhelm Arbenz das Schweizerische Tonkünstlerfest - der Vereinsvorstand wollte es wegen des Krieges absagen $^{3}$ - und veranstaltete in Biel ein kleines Fest, das in der Qualität geradezu internationales Niveau aufwies. ${ }^{4}$ Es muss auch Arbenz gewesen

1 Vgl. Gaffino/Lindegger: Bieler Geschichte, Bd. 2, S. 838-877, Kapitel »Frontenbewegung und Zweiter Weltkrieg (1933-1945)«.

2 Gemäß ausführlichen Auskünften meiner damals in der Bieler Uhrenindustrie tätigen Großeltern-Generation.

3 Merian: Fünfzig Jahre STV, S. 47.

4 In zwei Konzerten trat die Elite der Schweizer Interpretinnen und Interpreten in Biel auf, allen voran die Geigerin Stefi Geyer, die Cembalistin Silvia Kind, der Pianist Walter Frey sowie das Berner Streichquartett in der Gründungsbesetzung mit Alphonse Brun, Theo Hug, 
sein, der durchgesetzt hatte, dass ein Werk des Schweizer Juden Ernst Levy (1895-1981) gespielt wurde, ${ }^{5}$ den er als Klavierlehrer am Konservatorium Biel angestellt hatte. Allerdings konnte ihn Arbenz nicht lange in Biel halten. Ernst Levy wurde das zunehmend antisemitische Klima in der Schweiz unerträglich, er kündigte seine Stelle am Bieler Konservatorium ${ }^{6}$ und wanderte 1941 in die USA aus, wo er eine glänzende Karriere machte. Arbenz und das Bieler Konservatorium veranstalteten ihm zu Ehren am 27. Juni eine Abschiedsfeier. ${ }^{7}$ Im Jahresbericht wird Levy zudem eine flammende Hommage gewidmet, die in der Betonung des Schöpferischen und Unsystematischen als implizites Statement gegen den in Biel verbreiteten Antisemitismus zu begreifen ist. ${ }^{8}$

Ernst Levy, der mit Carl Seelig bekannt war und viel Lyrik von ihm vertonte (vgl. Kap. 11.2), könnte Wilhelm Arbenz auf Walsers Gedichte hingewiesen haben. Es ist nicht bekannt, wann und wo er auf diese gestoßen ist. ${ }^{9}$ Arbenz wurde nicht in Biel, sondern in Feuerthalen geboren; er wuchs in Zürich auf und studierte dort von 1918 bis 1923 Dirigieren und Schulgesang. Erst 1927

Walter Kägi und Richard Sturzenegger. Vgl. Ehinger et al.: Der Schweizerische Tonkünstlerverein, S. 322-324.

5 Interpretiert wurde das Werk von Silvia Kind, die speziell dafür nach Biel eingeladen wurde. Die Cembalistin Silvia Kind und der Komponist Roger Vuataz waren 1944 die einzigen Mitglieder des Tonkünstlervereins, die sich in einer tumultuösen Generalversammlung für den Dirigenten Hermann Scherchen einsetzten (vgl. Kap. 3, Anm. 11).

6 »Der prominente Pianist und Lehrer an der Musikschule, Ernst Levy, verlässt seine Stelle, um sich infolge der Zeitumstände nach Amerika zu begeben.« Lefert-Weibel: Bieler Chronik 1940 und 1941, S. 227.

$7 \gg$ An jenem tragischen Sonntagabend (22. Juni [1941, an dem das >Dritte Reich union angriff; RB]) scharten sich Direktorium, Vorstand, Schülerschaft und einige hiesige Freunde in einer kleinen Feier zum letzten Mal um den verehrten Lehrer und Meister.« [Musikschule Biel]: 10. Jahresbericht, S. 3 .

8 »Die Musikschule, die Musikschulgesellschaft, unsere Stadt verlieren in Ernst Levy einen eminenten Pianisten, einen Meister des Klaviers, der den Grössten unserer Tage beigesellt zu werden verdient. Wie spendete er Rat und Tat in seinen Klassenstunden, zeigte Wege, war unerschöpflich in guten Winken, stets herrlich unschematisch, den Jüngsten und Kleinsten ein ebenso aufrichtiger Rater und herzlicher Freund wie den zukünftigen Professionellen. Nur ein zutiefst schöpferischer Mensch konnte derart befruchtend wirken.« Ebd. S. 4. Levys Nachfolger in Biel wurde Adrian Aeschbacher aus »der fruchtbaren Aeschbacher-Dynastie« (Liebermann: Brief vom 10.08.1943). Vgl. Kap. 3.3, Anm. 10.

9 Auch in der bibliografisch nicht erfassten umfangreichen Korrespondenz, die im Archiv der Musikschule Biel erhalten ist und die die große Vernetzung von Wilhelm Arbenz mit der nationalen und internationalen Musikwelt belegt, ließ sich kein Hinweis finden. Ich danke dem Schulleiter Lionel Zürcher für den Zugang zu diesen bisher unbekannten Quellen im Konservatorium Biel. Den Schriftsteller kannte man in seiner Geburtsstadt kaum, was auch die Anekdote mit dem Schauspieler Alexander Moissi beweist, der 1920 im Bieler Stadttheater als Oswald in Ibsens Gespenstern gastierte und mit seiner stürmischen Begrüßung von Walser den Bielern bewusst machte, welch wichtiger Autor in ihrer Stadt lebte. Vgl. Mächler: Das Leben Robert Walsers, S. 143. 
kommt er nach Biel, als Robert Walser schon seit sieben Jahren in Bern lebt; wahrscheinlich hat er Walser nie persönlich getroffen. Arbenz stürzt sich förmlich ins Bieler Kulturleben, als er 1927 die Stelle als Musiklehrer am Bieler Progymnasium und Gymnasium erhält und sehr bald verschiedene Bieler Chöre dirigiert. Wie bei James Simon stammen die von Arbenz vertonten Texte alle aus dem 1909 erschienenen Band Gedichte, der 1919 in einer zweiten Auflage erschien.

\subsection{Unermüdlicher Förderer der Musik}

Wäre Walser dem engagierten Dirigenten und Musikerzieher einmal begegnet, hätte er ihm bestimmt ein Prosastück gewidmet und sich über seinen unermüdlichen Eifer lustig gemacht, mit dem er das Musikleben von Biel und Umgebung bestimmte. Arbenz war als bestens vernetzter und angesehener Mann mit besonderer Stellung in jeglicher Hinsicht das Gegenteil von Walser. Er begriff rasch, was der kleinen Industriestadt Biel fehlte: musikalische Bildung. Was Arbenz unternahm, um dieses Defizit zu beheben, würde man heute ein integriertes musikalisches Bildungskonzept nennen. Im Zentrum stand seine Stelle als Musiklehrer am deutschen Gymnasium, die er bis zu seiner Pensionierung 1963 innehatte. Bereits 1929 gründete er den Bieler Orchesterverein, der begabte Laienmusiker versammelte. $193^{2}$ rief er mit Freunden die Musikschule Biel ins Leben, die den Instrumentalunterricht professionalisierte und aus dem Dunstkreis privater Musiklehrpersonen herauslöste. Schon vier Jahre später wurden dort die ersten Berufsschulklassen eingeführt und die Musikschule wurde zum Konservatorium. Geschickt gelang es Arbenz immer wieder, angesehene Lehrkräfte ans Konservatorium Biel zu binden, auch wenn diese oft nur kurze Zeit blieben, bevor sie an größere Hochschulen wechselten. Arbenz war auch im Laienbereich aktiv und dirigierte alle wichtigen Bieler Chöre: die Bieler Liedertafel, den Lehrergesangsverein Biel, den Damenchor Concordia, die Berner Singstudenten, den Männerchor des Berner Liederkranzes und ab 195 o die Société chorale de Neuchâtel. ${ }^{10}$ Dazu übernahm er die Leitung des Bieler Stadtorchesters, eines Amateurorchesters, dessen Qualität er steigerte, indem er immer mehr semiprofessionelle Kräfte einband.

Mit diesen unterschiedlichen Ensembles interpretierte er zwar das große Oratorienrepertoire des 18. und 19. Jahrhunderts, aber er wusste auch, dass er ebenfalls das Volkstümliche pflegen musste. Als Komponist schrieb er für diese Chöre zahlreiche Lieder und Chorsätze, oft auf Dialekttexte.

Schuh et al.: Schweizer Musiker-Lexikon, S. 3 o. 
Nach 20 Jahren unermüdlichen Einsatzes hatte Arbenz in Biel so viel zur musikalischen Bildung der Bevölkerung beigetragen, dass er nun die besten Ensembles und Orchester nach Biel holen konnte und diese vor vollem Haus spielten. Anfänglich waren es Gastspiele des Berner Symphonieorchesters, bald aber schon große ausländische Orchester, Dirigenten und Solisten. Und auch die von ihm gegründete »Musikschul- und Konzertgesellschaft Biel-Bienne«, die eine Konzertreihe mit Gastspielen verantwortete, wurde zu einer Erfolgsgeschichte: In den knapp fünfzig Jahren ihres Bestehens waren die Konzerte fast immer ausabonniert.

In der ersten Hälfte des 20. Jahrhunderts gab es in vielen Schweizer Städten Figuren wie Wilhelm Arbenz: Erich Schmid realisierte Ähnliches in Glarus, Luzius Juon in Chur, Émile Jaques-Dalcroze in Genf - aber keiner hat es so erfolgreich wie Arbenz geschafft, den musikalischen Laienbereich, die Schulmusik, den Instrumentalunterricht, die Berufsausbildung, das städtische Orchester und einen internationalen Gastspielbetrieb auf- und auszubauen, sich also nicht bloß auf lokale Kräfte zu beschränken, sondern diese national und international zu vernetzen. Der Komponist und Musikkritiker Daniel Andres weist allerdings auch auf Probleme hin, mit denen sich Arbenz in der Bieler Provinz konfrontiert sah:

Biel zeigte sich in späteren Jahren nicht eben dankbar gegenüber diesem Kulturförderer, welcher der Stadt entscheidende Impulse gegeben hat. Der Dirigent Hermann Scherchen äusserte sich nach einer Probe von Arbenz mit dem Berner Stadtorchester: »Es hat mich gefreut, mit welcher Sachkenntnis und gründlichen Selbstvorbereitung Sie dem Orchester gegenübertraten.«Und ein anderer bedeutender Dirigent, Felix Weingartner, meinte sinngemäss, wenn man junge Leute wie Arbenz mit dem Orchester arbeiten sehe, so müsse einem um den Dirigenten-Nachwuchs in der Schweiz nicht bange sein. [...] In Biel hingegen hat man Arbenz schon in den frühen Fünfzigerjahren als Orchesterdirigent kaltgestellt. ${ }^{11}$

Die industrielle Entwicklung der Stadt Biel kam den musikalischen Bemühungen von Arbenz entgegen: Die Einwohnerzahl war massiv angestiegen; bei der Volkszählung von 1964 werden fast 65 ooo Einwohner verzeichnet. Zu diesem Zeitpunkt hat Arbenz bereits mit schweren psychischen Problemen zu kämpfen, die dazu führen, dass man ihm die Kündigung und den Rückzug aus den vielen Ämtern nahelegt. Der Tod seiner Frau, der französischen Steinbildhauerin Denise Chenot (1898-1964), verstärkte Arbenz' Erkrankung. Am längsten hielt sich Arbenz als Konservatoriumsdirektor; die Situation

11 Andres: Musik, ein Leben lang, S. 53 f. 
eskalierte 1965, sodass er auch von dieser Aufgabe definitiv entbunden wurde. 1969 verstarb Wilhelm Arbenz in Biel.

\subsection{Drei Lieder}

Die drei Walser-Lieder bilden in Arbenz' kompositorischem Gesamtwerk zusammen mit den Liedern auf Texte von Josef Viktor Widmann und Hermann Hesse - ein spezielles Repertoire, das sich in Qualität und Kunstanspruch von den zahlreichen Volksliedern, Männer- und Frauenchören, historischen Sing- und Festspielmusiken, die Arbenz sonst komponiert hat, deutlich unterscheidet. Rückblickend ist es bedauerlich, dass Arbenz nicht mehr solche Werke komponierte, die sich dem Zeitgeist entziehen und nicht an den musikalischen Fähigkeiten von Laien orientieren.

Schon die Auswahl der drei Gedichte ist auffällig. In ihrer steigenden Kadenz von Enttäuschung, Trug und Befriedung in der Wintersonne bilden sie eine schöne Einheit. Wegen der Bedeutung des kurzen Liederzyklus in der musikalischen Robert Walser-Rezeption, seien im Folgenden die beiden ersten Lieder ausführlicher betrachtet.

Enttäuschung ist ein vertracktes Gedicht, das syntaktisch aus zwei Sätzen besteht und mit einer quasi dilettantischen Reim- und Rhythmusstruktur spielt: zwei homonyme Reime (nie - nie sowie ist - ist), ein überlanger zweiter und ein verschachtelter dritter Vers, letzterer ausgerechnet, um das Reimwort »sie« zu erreichen.

Enttäuschung vergißt man nie, wie der Lockruf des Glücks unvergeßlich ist.

Erinnern ist Sehnsucht, ach, sie, weil sie so unermeßlich ist, vergißt man nie. (SW 13, 24)

Wilhelm Arbenz benützt in allen drei Liedern eine freitonale und fast impressionistische Musiksprache, die durch chromatische Linien und Gegenbewegungen bestimmt ist und teilweise extrem dissonante Klänge zulässt. Es gibt keine tonartliche Bindung mehr, entsprechend verzichtet Arbenz auch bei allen Liedern auf Anfangsvorzeichen. Zwar kommen tonale Klänge - vor allem Sept- und Nonakkorde - vor, aber diese werden impressionistisch verwendet, nicht aufgelöst und in keine tonartliche Logik eingebunden. Ein wichtiges Charakteristikum sind die weiten und die tiefen Lagen, die architektonisch, aber auch den Text deutend eingesetzt werden. So wird gleich zu Beginn das Tritonus-Intervall der Sängerin auf »Enttäuschung« vom Klavier 


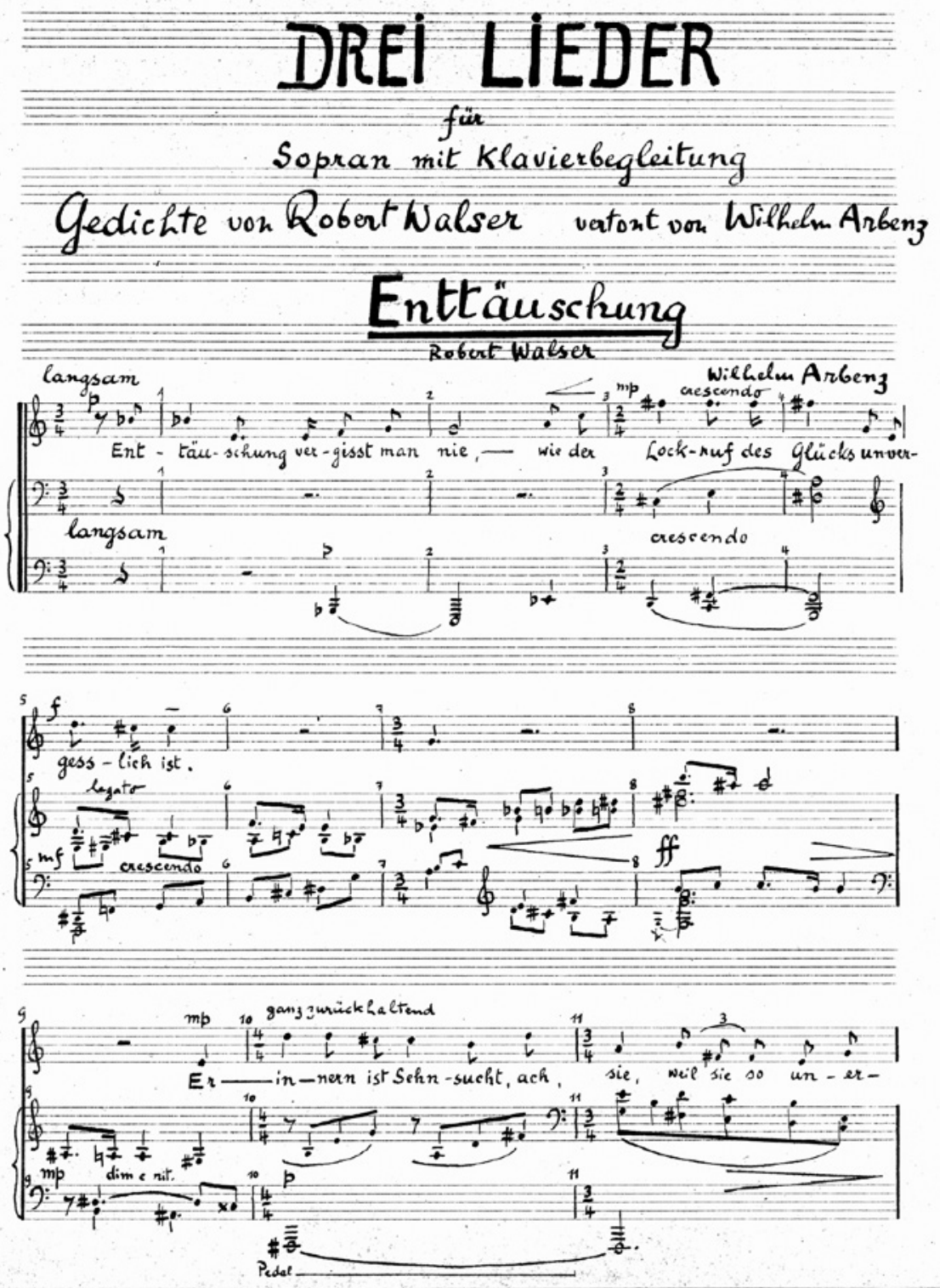

Abb. 3 Wilhelm Arbenz: Enttäuschung und Trug aus Drei Lieder nach Robert Walser 

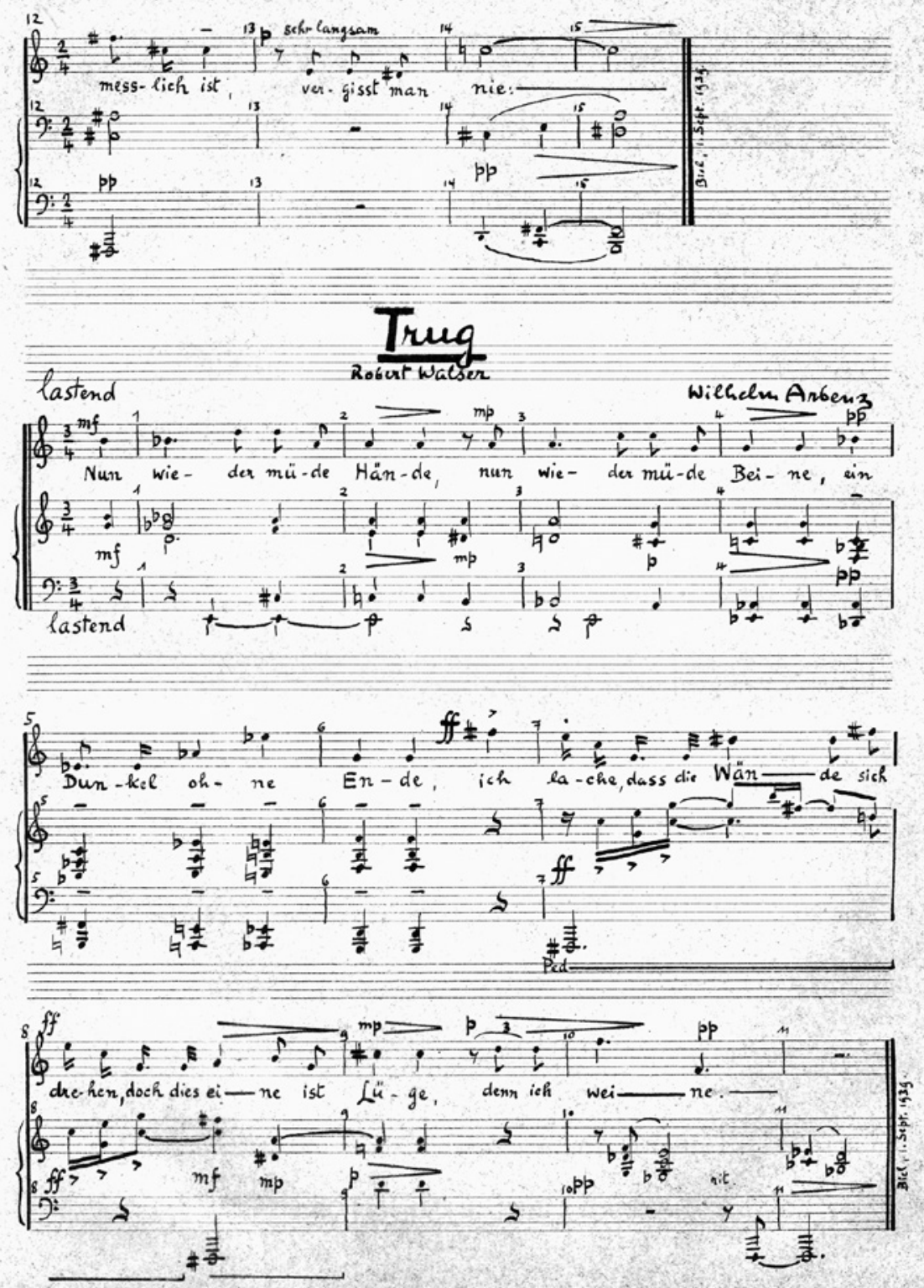
drei Oktaven tiefer wie ein Schatten imitiert: ein symbolisches Zeichen für das Nichtvergessenkönnen. Beim »Lockruf des Glücks« wird der Satz auf dreieinhalb Oktaven gespreizt; harmonisch erklingt auf »Glücks« ein Septakkord auf $h$ (in der Gestalt, aber nicht in der Funktion eines Dominantseptakkords), der ganz am Schluss des Liedes als Septnonakkord nochmals erklingt und das Nichtvergessen des Glücks entsprechend heraushebt.

Zwischen die beiden Sätze des Gedichts setzt Arbenz ein Zwischenspiel, das fast nur aus chromatischen Fortschreitungen besteht, die sich in Takt 7 auf den Umfang einer Sexte zusammenziehen, um gleich darauf in Takt 8 in einen übermäßigen Nonakkord zu explodieren. Während der erste Teil in der Gegensätzlichkeit von introvertierter Enttäuschung und mit großen Intervallsprüngen gesungenem Lockruf geradezu expressionistisch vertont wird, wechselt Arbenz beim dritten Vers den Stil der Vertonung. Er begegnet Walsers hinkender Deklamation mit einem Parlando-Ton. Zwar hallt in der Tiefe immer noch das $F i s_{1}$, aber darüber erklingt in Takt 10 ein fröhliches D-Dur im Viervierteltakt. Mitten im Wort »unermesslich « wird diese unschuldige Welt nach Fis-Dur und zugleich in die weiteste Lage des ganzen Liedes gerissen: 4 Oktaven. In Takt 13 setzt die Stimme wie zu Beginn ohne Begleitung ein, und das Lied endet - unerlöst - im schon erwähnten, dissonant-offenen Klang.

Beim zweiten Lied wählt Arbenz für die Singstimme in den ersten drei Versen von Trug ( $\mathrm{SW} \mathrm{13}_{13}$ 28) einen einfachen periodischen Liedaufbau, der das Schlichte des Gedichts einfängt. Erstaunlich allerdings sind die Harmonien, die das Klavier - im gleichen Rhythmus - dazu spielt. Da entstehen teilweise scharfe Dissonanzen und beim fünfstimmigen Klaviersatz in Takt 5 treibt die Bewegung völlig auseinander, weil die linke Hand in Quinten ab- und die rechte Hand mehr oder weniger chromatisch aufsteigt. Wie ein hysterischer Ausbruch ist das »ich lache « vertont; gleichsam alles verrutscht: Von b-Vorzeichen wird auf Kreuze gewechselt, Klaviersatz und Singstimme laufen in Gegenbewegung ineinander und überkreuzen sich hier zum ersten Mal in diesem Liederzyklus. Arbenz vertont sehr präzis, denn er baut die verrückte Stelle nicht überdimensioniert aus, sondern nimmt bei »ist Lüge« den rhythmischen Modus des Anfangs wieder auf. Von einem Fortissimo bei Takt 8 wird in ein Pianissimo bei Takt 10 zurückgeblendet. Feinsinnig und ohne Pathos wird das »denn ich weine « musikalisch gefasst: zuerst »denn ich « leicht verzögert und zu Triolen verkürzt, dann der schlichte und in diesen drei Liedern einzige Oktavfall der Singstimme auf »wei-ne«.

Das dritte Lied mit dem Glanz und Kälte verbindenden Titel Wintersonne ist ganz anderer Natur. 


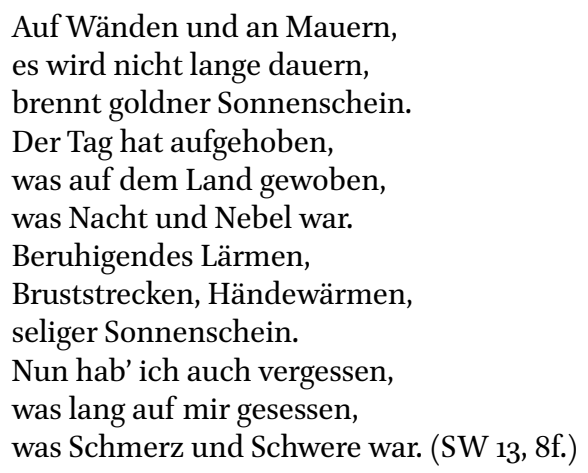

Die Musik zu diesem die Beruhigung und innere Befriedigung ankündigenden Gedicht weist einen dichten, wegen seines Umfangs von knapp sieben Oktaven teilweise auf drei Systemen notierten Klaviersatz auf, in dessen Mitte - und nicht wie in den ersten beiden Liedern meistens darüber - sich die Singstimme befindet. Insbesondere die dritte und die viertletzte Zeile rund um das Wort »Sonnenschein « sind hochmelismatisch und in expressionistischer Überspanntheit vertont.

\section{$2.4 \quad$ Rezeption}

Die drei Lieder wurden am »Pfingsttagung « genannten Tonkünstlerfest am 1. Juni 1941 im Saal der Società Elettrica in Locarno uraufgeführt. ${ }^{12}$ Gesungen wurden die Lieder von Lucia Corridori, ${ }^{13}$ begleitet von Walter Lang. ${ }^{14}$ Karl Heinrich David betont in der Schweizerischen Musikzeitung, dass Corridori und Lang »die heiklern modernen Lieder in bestes Licht stellt[en]«, während die übrigen Gesänge von anderen Interpreten dargeboten wurden (Elsa Scherz-Meister und Leopoldo Casella). Die Liedervorträge hatten bei David keinen besonderen Eindruck hinterlassen:

12 Ehinger et al.: Der Schweizerische Tonkünstlerverein, S. 324f. Aufgrund einer falschen Auskunft habe ich im Robert Walser-Handbuch die Uraufführung fälschlicherweise auf das Tonkünstlerfest 1937 in Lugano datiert. (RWH, 391)

13 Lucia Corridori (1899-1976) war eine vielgefragte Koloratursopranistin und Gesangsprofessorin am Konservatorium Luzern.

14 Walter Lang (1896-1966) war Komponist, Kapellmeister, Pianist und Klavierprofessor an den Konservatorien Basel und Bern sowie an der Musikakademie Zürich. 
Im zweiten Konzert waren die Liedgruppen (wie meist an Tonkünstlerfesten) das Schwächste. Am meisten interessierten noch die modern getönten, musikalischen Aquarelle von Wilhelm Arbenz (nach Texten von Robert Walser) und effektsichere, neuromantische Gesänge des Baslers Ernst Müller. ${ }^{15}$

In der Neuen Zürcher Zeitung schreibt er noch kürzer: »Lucia Corridori lieh feingetönten Liedern von Wilhelm Arbenz [...] ihren beseelten Vortrag.«16

Bei aller Beiläufigkeit, mit der die drei Lieder von David erwähnt werden, mussten sie im Kontext der damaligen Zeit doch als »modern« aufgefallen sein. Acht Jahre später lässt Willi Schuh in seinem Aufsatz zur Deutschschweizer Musik die Modernität dieser Lieder unerwähnt und notiert nur allgemein, Arbenz sei »mit kleineren Chorwerken für gemischten, Männer- und Frauenchor, Liederzyklen, einer hübschen Schulkantate >Jugend im Schneeく (Text von Georg Thürer), und einem Bieler Festspiel als Komponist hervorgetreten. «17 Damit verschwand die feinsinnige Kunst seiner Walser-Lieder in der Aufzählung von Arbenz' Kompositionen zur geistigen Landesverteidigung. Weitere Aufführungen zu Lebzeiten des Komponisten konnten nicht nachgewiesen werden. ${ }^{18}$

\footnotetext{
15 David: Tonkünstlerfest in Locarno, S. 189.

16 David: Schweizerischer Tonkünstlerverein, Blatt 2.

17 Schuh: Die Musik in der alemannischen Schweiz, S. 220.

18 Wieder aufgeführt wurden die Lieder durch Luisa Castellani und Pierre Sublet an der Holliger-Walser-Woche 1996 in Biel, 57 Jahre nach ihrer Niederschrift 1939. Indiz dafür, dass sie in der Zwischenzeit nicht aufgeführt wurden, ist $u$. a. die Tatsache, dass Fehler nicht eliminiert wurden, z. B. die irrtümlich notierte Note in der Singstimme im ersten Lied, Takt 7 .
} 